\title{
Relaciones públicas y Juego de Tronos: la imagen del Sevilla Fútbol Club, S.A.D. tras la visita de los actores al Sánchez Pizjuán
}

\section{Harreman publikoak eta Game of Thrones: Sevilla Fútbol Club, S.A.D. taldearen irudia aktoreek Sánchez Pizjuán zelaia bisitatu ondoren}

\section{Public relations and Game of Thrones: the image of Sevilla Fútbol Club, S.A.D. after the visit of the actors to Sánchez Pizjuán}

\section{Juan Pablo Micaletto Belda'}

Rafael Cano Tenorio²

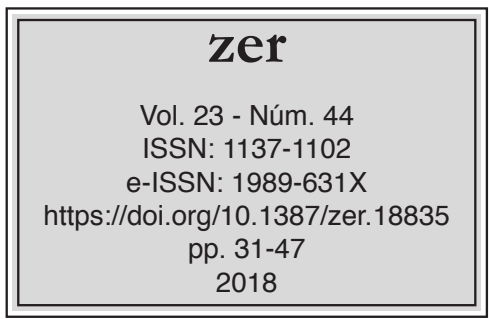

Recibido el 30 de noviembre de 2017, aceptado el 8 de enero de 2017.

\section{Resumen}

El artículo versa sobre la acción de publicity desarrollada por el Sevilla Fútbol Club, S.A.D. con la visita de los actores de Juego de Tronos al estadio Sánchez Pizjuán. En la investigación se analizan los contenidos generados por el club en las redes sociales Twitter e Instagram, y la gestión informativa realizada por la prensa online a raíz de este acto, con la hipótesis principal, confirmada, que esta acción adquirió efectos positivos en la imagen corporativa del club.

Palabras clave: Relaciones públicas; imagen corporativa; publicity; redes sociales; Juego de Tronos; fútbol.

\section{Laburpena}

Game of Thrones (Tronuen Jokoa) serieko aktoreek Sánchez Pizjuán futbol zelaira eginiko bisitaren harira Sevilla Fútbol Club, S.A.D. taldeak gauzatutako publizitate-ekintzari buruzkoa da artikulua. Ikerketan, taldeak Twitter eta Instagram gizarte-sareetan sortutako edukiak eta ekitaldiaren harira online prentsan eginiko informazio-kudeaketa aztertzen dira.

Centro Universitario San Isidoro (Universidad de Pablo de Olavide),jmicaletto@ centrosanisidoro.es

2 Universidad de Cádiz, lete@jerez.es 
Azterlanaren hipotesi nagusia da (baieztatua), ekimenak eragin positiboa izan zuela taldearen irudi korporatiboan.

Gako-hitzak: Harreman publikoak; irudi korporatiboa; publicity; sare sozialak; Game of Thrones; futbola.

\section{Abstract}

The article addresses the Sevilla Fútbol Club S.A.D.'s advertisement campaign during the visit of 'Game of Thrones' stars to the Sánchez Pizjuán stadium. The research analyzes the contents generated by the club on Twitter and Instagram social media, and the information management carried out by the online media as a result of this act. The main hypothesis, confirmed, is that this action provided positives effects on the club's corporate image.

Keywords: Public relations; corporate image; publicity; social media; Game of Thrones; football. 


\section{Introducción}

En la sociedad digital, las acciones de relaciones públicas son empleadas por las organizaciones para difundir ante sus públicos una imagen adecuada a sus fines y actividades. Estas acciones tienen como valor principal fortalecer la imagen corporativa de las organizaciones, mejorando su posicionamiento y favoreciendo su desarrollo social y empresarial.

Capriotti (1999: 15) entiende que la imagen corporativa es aquélla que tienen los públicos acerca de una organización en cuanto entidad como sujeto social, y a la idea global que tienen sobre sus productos, sus actividades y su conducta. Villafañe (2005: 112) explica la importancia de la misma como parte fundamental del concepto dimensión estratégica, que requiere la adopción de un posicionamiento reputacional. En este sentido, una empresa pretende influir en la mente de sus stakeholders, como el resultado de una relación eficaz con los mismos con objeto de mejorar su reputación.

Una de las técnicas utilizadas en el ámbito de las relaciones públicas consiste en desarrollar acciones de publicity, capaces de generar notoriedad y suscitar el interés de los medios de comunicación. Éstos tienen una gran importancia para el conjunto de las organizaciones, debido a su cobertura y a su capacidad de segmentación; pues "los medios de comunicación se dirigen tanto a un público general como a uno especializado" (Castillo, 2010: 106).

A este hecho se suma que las redes sociales están actuando como plataformas difusoras de noticias, informaciones y valoraciones, que son de gran interés para el conjunto de las organizaciones deportivas (Cole, 2016: 15). Las redes sociales permiten elaborar, compartir y consumir contenidos generados tanto por las organizaciones como por el resto de usuarios. Por esta razón, estas herramientas son de gran interés para las identidades deportivas (Lobillo y Muñoz, 2016: 199), que permiten interaccionar, de manera directa, con sus diversos públicos.

El día 7 de noviembre de 2016, el departamento de comunicación del equipo español Sevilla Fútbol Club, S.A.D. desarrolló una técnica de relaciones públicas basada en la publicity, que consiste en desarrollar un evento noticiable que atraiga la atención de los medios de comunicación (Cameron, Wilcox y Xifra: 2009). Esta acción se desarrolló a través de los medios de comunicación y las redes sociales. El equipo de la ciudad hispalense organizó una visita guiada a los actores de la serie de televisión Juego de Tronos al estadio Sánchez Pizjuán, para presenciar un partido de fútbol que enfrentaría al Sevilla con el Fútbol Club Barcelona.

Juego de Tronos es uno de los programas de televisión más populares en la actualidad, con más de 8 millones de televidentes en su última temporada, según la British Broadcasting Corporation, siendo además, la serie de ficción de mayor nivel de interés mediático y seguimiento en redes sociales a nivel mundial. Además, según la misma fuente, acumula la mayor cantidad de premios Emmy, uno de los reconocimientos más prestigiosos de programas televisivos a nivel internacional.

A su llegada al estadio, miles de fans tararearon el theme song -tema musical de la serie con el que empieza un determinado programa-, ante la expectación del reparto, ubicada en uno de los Palcos VIP del estadio, que había sido decorado al detalle con elementos de la serie. Además, cada uno de los actores recibió una camiseta 
personalizada del Sevilla con sus nombres y una bufanda del equipo que llevaron durante todo el encuentro.

La visita de los actores de la serie al Sánchez Pizjuán se convirtió en un evento de interés mediático. Con el paso de las horas, tanto la prensa convencional como la especializada se hicieron eco de este acontecimiento, publicando decenas de noticias sobre el acto, adaptadas a diferentes formatos -televisión, radio, prensa e internet-. El Sevilla, al igual que los actores de la serie, retransmitieron el evento a través de las redes sociales, compartiendo vídeos, fotografías y comentarios relacionados con la visita.

El objeto de esta investigación posee dos partes: el análisis de los contenidos generados por el club a través de las redes sociales y el estudio de la gestión informativa realizada por la prensa online a raíz de este acto, con el objetivo principal de medir los efectos que esta acción de publicity tuvo en la imagen corporativa del Sevilla. La hipótesis principal fue que esta acción de relaciones públicas permitió al club hispalense proyectar una imagen favorable ante sus públicos, generando impactos favorables en los medios de comunicación.

\section{Marco teórico}

\subsection{Comunicación deportiva en el entorno digital}

Los estudios sobre la comunicación deportiva en Internet han aumentado en la comunidad científica de manera considerable en los últimos años. Esta tendencia al alza resalta el interés social que adquiere esta forma de comunicación en sus diferentes vertientes de estudio. Por esta razón, Alonso y Avalos (2013: 40) señalan que "el estudio de los medios de comunicación (media studies) y del deporte como objeto de estudio en el campo de la comunicación son dos tendencias de investigación que no han dejado de crecer".

La imagen y la reputación constituyen los activos más importantes de las organizaciones, públicas o privadas, ya que éstas se deben principalmente a la imagen que generan ante sus públicos. Paché e Ika (2017: 34) explican la reciente transformación que se está produciendo en los clubes de fútbol en materia de marketing y relaciones públicas, que ha hecho que los responsables de comunicación presten una especial atención a todos los elementos que influyen en la comunicación corporativa de los equipos.

Internet constituye una importante plataforma de comunicación para el deporte. Por esta razón, Moragas (2003: 26) explica que "los medios de comunicación ofrecen una plataforma de exposición para el deporte, lo que constituye la publicidad gratuita necesaria para promover la participación de un mayor número de deportistas, espectadores y consumidores".

La comunicación deportiva en los entornos digitales destaca principalmente por su bidireccionalidad. Sobre esta dinámica comunicativa, Domínguez, Álvarez y Martí (2012: 51) resaltan que "con la digitalización se han abierto más canales de respuesta en la comunicación organizacional acercándose a la bidireccionalidad comunicativa de la que habla el situacionismo y el funcionalismo comunicológico". No obstante, estos autores señalan que sigue primando la gestión de la imagen, la publicidad y la relación con los medios de comunicación. 
De Casas, Rodríguez y Aguaded (2017: 147) explican que con el auge de las nuevas tecnologías, la prensa digital ha adquirido un rol notorio a la hora de informar sobre acontecimientos relacionados con el deporte, que pueden generar discursos persuasivos.

\subsection{La web 2.0 y las redes sociales como herramientas de marketing deportivo}

Según Coutinho y Luzzi (2017: 24), es posible mediante el uso de la comunicación la promoción de productos y servicios usando redes sociales y sitios web de clubes deportivos para interactuar con los aficionados o seguidores antes y durante los partidos, eventos o actividades dentro de la arena. La web 2.0 tiene diversas características destacables, "instantaneidad, multimedialidad, personalización e interactividad aparecen como los rasgos definitorios de los cibermedios deportivos en la comunicación 2.0" (Favaretto, 2014: 239), que determinan el funcionamiento de esta clase de páginas.

Las nuevas tecnologías suponen un elemento relevante para la comunicación deportiva. Cancelo y Almansa (2013: 424) señalan que "las nuevas tecnologías han aportado rapidez y comodidad y han abierto un abanico de posibilidades impensables. En este sentido, Internet ha supuesto grandes ventajas y oportunidades para quienes gestionan la comunicación". Según Cole (2016: 2), las redes sociales no son un concepto nuevo, pues sus raíces pueden remontarse a los principios de la tecnología de la web 2.0. Sobre la utilidad de las redes sociales para los clubes de fútbol profesionales, Lobillo y Muñoz (2016: 197) exponen:

Como medio propio de los clubes de fútbol, las redes sociales son muy útiles, como en cualquier otra organización, por lo que se utilizan como herramienta de comunicación organizacional. Las nuevas tecnologías son constantemente usadas por las organizaciones deportivas, ya que éstas les permiten interactuar con sus públicos de una forma inmediata y eficaz: los medios de comunicación, los accionistas, los peñistas, los aficionados, patrocinadores, anunciantes, etc.

Las redes sociales suponen una parte importante del trabajo de los departamentos de comunicación de los clubes profesionales de fútbol. Sobre la importancia de la presencia por parte de clubes de fútbol profesionales en las redes sociales, Castillo, Fernández y Castillero (2016: 251) señalan:

Los clubes de fútbol con mayores ingresos del mundo desarrollan una estrategia de comunicación 2.0 en la mayor parte de las redes sociales existentes. Algunos de los clubes hicieron su adaptación casi desde el inicio de la red como es el caso de Youtube que comenzó en febrero de 2005 y en ese mismo año tres clubes ya disponían de su propio canal como son Liverpool, AS 
Roma e Inter de Milán. En general, muestran una adaptación adecuada a la creación de las redes sociales y a las plataformas de la comunicación digital.

Facebook es la red social que aglutina a más seguidores y simpatizantes de los clubes profesionales de fútbol, por delante de Twitter, Instagram, Youtube y Google Plus. "Por lo que se refiere a la presencia de clubes en la red social líder en el mercado (Facebook), el conjunto de los equipos analizados sumaban cerca de 50 millones de fans en ella a finales de enero de 2012" (Sotelo, 2012: 223). Además, según Cano (2017: 55), es la red social con mayor nivel de emisión de porcentaje de contenido relacionado con marketing, por parte de los clubes de fútbol de élite.

Para Cole (2016: 15), los medios sociales, y en particular Facebook, han experimentado una prominencia en la práctica de marketing contemporánea en los últimos diez años. En el deporte, las redes sociales son reconocidas como una efectiva herramienta de marketing, ya que puede aportar ventajas únicas para vender un producto deportivo. Por su parte, la red social Instagram, según Rojas y Panal (2017), tiene unas funcionalidades propias que facilitan la interacción entre sus usuarios, algunas compartidas con otras redes sociales y otras genuinas, que tienen gran utilidad para el periodismo.

Las redes sociales suponen un medio que da facilidades para llegar a los públicos de las organizaciones. Al respecto, Lobillo y Muñoz (2016: 199) apuntan:

Las redes sociales posibilitan a los clubes llegar con facilidad a un público bastante importante, no solo por cantidad, sino también por su fidelidad. Para comunicarse con estos públicos, principalmente los aficionados (aunque no son los únicos focos de atención de los clubes profesionales), estas entidades poseen perfiles en las principales redes sociales. De forma que los clubes de fútbol ya han instaurado, de manera más o menos uniforme, los canales sociales como herramientas de comunicación.

\subsection{Públicos de los clubes de fútbol}

Según Capriotti (2009), la noción de Público es uno de los pilares básicos en el ámbito de la Comunicación Corporativa, las Relaciones Públicas, la Publicidad, el Marketing y el Management, y se lo reconoce como tal, poniéndose de manifiesto por parte de investigadores y profesionales como un concepto de vital importancia para las organizaciones.

La relación de los clubes profesionales de fútbol con sus públicos debe ser tenida en cuenta de manera importante por los encargados de la gestión de la comunicación. Olabe (2010) señala que los departamentos de comunicación de los clubes de fútbol profesionales conceden importancia a realizar acciones comunicativas dirigidas a los públicos externos, internos e intermedios, contando con los medios de comunicación como el público más importante de los clubes profesionales de fútbol. En este sentido, según este autor, los gabinetes de comunicación de los 
clubes profesionales de fútbol buscan condicionar la agenda y las rutinas de los medios de comunicación a los que se dirige, utilizando de manera destacada las categorías de contenido relacionadas con ruedas de prensa, comunicados oficiales, entrevistas, reportajes y uso de la web oficial, con actualización periódica de contenidos dentro de la misma.

Olabe (2012: 280) explica que la aparición de las nuevas tecnologías ha introducido numerosos cambios:

Las relaciones entre las entidades deportivas, los medios de comunicación y las audiencias de esos deportes se han modificado con la aparición de la web 2.0 y sus plataformas sociales, donde las audiencias, ya sean socios, aficionados, seguidores u otros públicos de las organizaciones deportivas no solo interactúan, sino que también actúan como generadoras de contenidos relacionados con esas entidades.

Los clubes profesionales de fútbol se relacionan en el entorno digital con grupos diferentes de receptores. Sobre la repercusión de emitir mensajes a grandes grupos de personas receptoras, Cancelo y Almansa (2013: 426) señalan:

En definitiva, la universalización de la posibilidad de ser emisor de mensajes que lleguen a grupos numerosos a través de Internet ha provocado la ruptura del esquema tradicional de emisor-receptor. Es el caso de las redes sociales. Por el volumen de participantes y por las condiciones de control sobre el mensaje, parecen una herramienta idónea para ganar adeptos y para fomentar la relación entre las universidades y sus públicos, que ahora sí que puede ser directa.

\section{Metodología}

El trabajo muestra un estudio de caso abordado a través del método hipotético inductivo, compuesto por dos objetivos secundarios:

- El análisis cualitativo de diversas noticias, en prensa online, relacionadas con la acción de publicity desarrollada por el Sevilla Fútbol Club.

- El estudio de los contenidos generados por el Club por medio de las redes sociales a raíz de este acto.

En el desarrollo del artículo se utilizaron fuentes secundarias. Dichas fuentes han permitido analizar qué efectos generaron en la prensa la visita de los actores al Sánchez Pizjuán. El contenido analizado en la prensa ha sido extraído a través del buscador Google News por medio de las siguientes palabras claves: Juego de Tronos 
en Sevilla, fútbol y Juego de Tronos y actores de Juego de Tronos en el Sánchez Pizjuán. En su conjunto, han permitido analizar de una forma amplia y variada la repercusión de este evento, tanto en prensa especializada como no especializada.

La selección muestral fue intencional y teórica. Se realizó detectando los contextos de unidades de análisis relevantes para el problema de investigación y conduciendo la selección hacia su significación para los objetivos de la investigación. De esta forma, las noticias que componen la muestra han sido seleccionadas en función a la conexión que mantienen con el objeto de la investigación y a los objetivos presentados. El modo de recopilar toda la información fue a través de un vaciado de prensa y un análisis de contenido cualitativo sobre el evento.

Dentro de las publicaciones analizadas, siguiendo el método de análisis de Micaletto y Gallardo (2015: 98) utilizado para analizar la crisis institucional del ébola en España, se ha contemplado el estudio de los siguientes elementos:

- Cuerpo

- Entradillas

- Fotografías

- Titulares

- Vídeos

Estos elementos han permitido extraer los encuadres noticiosos de la prensa, evaluando cualitativamente los mensajes periodísticos publicados sobre la visita de los actores al Sánchez Pizjuán. Dentro de esta muestra se han seleccionado aquellas publicaciones que poseen algún contenido sobre esta acción de relaciones públicas y se les ha asignado un valor positivo, negativo o neutro en función de cómo repercuten en la imagen y reputación del club.

Se consideran noticias positivas a aquellas publicaciones que describen la visita de los actores al Sánchez Pizjuán con un vocabulario favorable y transmiten una imagen positiva de la iniciativa organizada por el club. Por el contrario, se establece como publicaciones negativas aquellas que describen con un vocabulario desfavorable dicha iniciativa. Finalmente, aquellas informaciones que arrojan tanto datos favorables como desfavorables han sido catalogadas como neutras.

Además, se han analizado las publicaciones realizadas por el club de fútbol en las redes sociales Instagram y Twitter, siguiendo el modelo de Cano (2018). Por otro lado, se ha analizado el tipo y la cantidad de contenido que se ha generado desde cada cuenta oficial, estableciendo previamente un cuestionario que permite trasladar el objetivo de averiguar los tipos de contenido elaborados por el club de fútbol en cada cuenta en variables, y las variables a indicadores. Las variables en este caso se clasificarán en la tipología del contenido que tiene cada elemento publicado que se comparte en las redes sociales analizadas.

El acceso a las direcciones oficiales de las redes sociales se ha obtenido a partir de la web oficial del Sevilla. Las redes sociales analizadas son Instagram y Twitter, 
ya que dichas redes sociales presentan, de manera diferente, contenidos relacionados con el objeto analizado en las fechas de análisis. Las cuentas oficiales del club en cada red social analizada son: “@ sevillafc” y "SevillaFC”, respectivamente. Dichos medios, además, permiten extraer para el estudio, de manera fiable, los contenidos publicados en las fechas de análisis. Se ha considerado que los contenidos en la red social Facebook no ofrecían fiabilidad y por tanto carecían de validez para su estudio científico, ya que sólo se permite acceder a los contenidos más destacados según el algoritmo de la red social.

La investigación se ha realizado entre el 4 y el 8 de noviembre 2016, coincidiendo con el día en el que los actores asistieron al partido (6 de noviembre de 2016) y los días anteriores y posteriores al mismo. El contenido publicado de cada red social se ha clasificado en fichas de análisis de las diferentes redes examinadas en las siguientes clases y categorías de contenido, siguiendo la clasificación de la investigación realizada por Cano (2018), y añadiendo variantes relacionadas con el actual estudio:

- "Juego de Tronos": Publicaciones que ha realizado el club de fútbol y que guardan relación con la asistencia al estadio de los actores protagonistas de la serie. Se incluye la previa de su estancia en Sevilla, y la posterior repercusión que tuvo a nivel mediático.

- "Institucional": Contenido relacionado con publicaciones referidas a informaciones que tienen relación con actos o con noticias que informan sobre cuestiones derivadas de la directiva, relaciones con otros clubes, participación en campañas con la imagen del club, relaciones con otras empresas o instituciones públicas o privadas, declaraciones públicas del presidente o directivos, actos con las peñas, imagen del club de fútbol, responsabilidad social corporativa, actos de la fundación, publicaciones relacionadas con el estadio o sede, acontecimientos históricos o efemérides del club de fútbol, y pésames por personajes ilustres relacionadas con la institución.

- "Agenda": Publicaciones que hacen referencia a información relacionada con el día a día del club, los entrenamientos u organización del trabajo de las plantillas de los clubes de fútbol, incluyendo jornadas de descanso -en el caso de no haber entrenamiento- del primer equipo del club de fútbol. En esta categoría de contenido se incluye el contenido relacionado con la información sobre la enfermería y lesionados de la plantilla del club.

- “Competición": Publicaciones y contenido que se genera en relación a los partidos en los que compite el Sevilla.

- “Jugadores": Publicaciones referidas a información relacionada con los miembros de la plantilla. Incluye información sobre convocatorias internacionales, firmas y actos públicos, premios individuales que reciben los jugadores o miembros del cuerpo técnico, y datos sobre estadísticas o especiales sobre los mismos. 
- "Cantera": Publicaciones y contenidos referidos a los equipos y jugadores de la cantera o academia de formación del club de fútbol. Incluye información relacionada con entrenamiento, pre-partido, partido, postpartido, rueda de prensa, entrevistas, jugadores y fichajes.

- "Rueda de prensa": Publicaciones relacionadas con todo lo que sucede en la sala de prensa del estadio o sede de entrenamiento del club de fútbol durante el pre-partido y post-partido. Se incluye en esta categoría de contenido las declaraciones que se producen por parte de los miembros de plantilla y cuerpo técnico del club de fútbol en la zona mixta y zonas de entrenamientos.

- "Entrevistas": Categoría de contenido que incluye publicaciones alusivas a otras declaraciones que no se dan en la anterior categoría. Incluye entrevistas a través de redes sociales.

- "Afición": Categoría de contenido que incluye contenido y publicaciones referidas a la venta o información de las entradas de un partido a disputar por el club de fútbol, los desplazamientos para acompañar al equipo a un partido como visitante. Se incluye contenido multimedia y agradecimientos a los seguidores.

- "Patrocinadores": Se incluye en esta categoría de contenido todas las publicaciones relacionadas con patrocinadores, sponsors y compromisos comerciales del Sevilla.

- "Otros medios": Contenidos que enlazan con otros medios oficiales del Sevilla. Se incluye en esta categoría promoción de los mismos.

- "Retweet": Esta categoría de contenido se refiere a la categoría de publicaciones que son copia de otros contenidos de una página web, otro usuario u otro tweet. Esta categoría sólo existe en el caso de análisis de las categorías de contenido en las cuentas oficiales en la red social Twitter. En el resto de redes sociales analizadas o en las salas de prensa virtuales no aparece como categoría de herramienta de comunicación.

\section{Resultados}

\subsection{Noticias online}

Tras las múltiples búsquedas realizadas mediante el buscador de noticias Google News, se han seleccionado las siguientes noticias junto a sus respectivos titulares, facilitando que cualquier persona pueda consultarlas a fin de comprobar los datos obtenidos. Estas fuentes han sido localizadas y clasificadas en diferentes medios de comunicación, permitiendo observar con determinación la repercusión de este evento. 
Tabla 1. Resultados de noticias localizadas en prensa generalista.

\begin{tabular}{|c|c|c|}
\hline Diarios & Titular & $\begin{array}{l}\text { Impacto en la } \\
\text { imagen del club }\end{array}$ \\
\hline $\begin{array}{l}\text { Libertad } \\
\text { digital }\end{array}$ & $\begin{array}{l}\text { Los actores de Juego de Tronos se } \\
\text { divierten en el Sánchez Pizjuán }\end{array}$ & Positivo \\
\hline$A B C$ & Los actores de Juego de Tronos, en el Reino de Nervión & Positivo \\
\hline El Mundo & $\begin{array}{l}\text { Leo Messi deja alucinados a los actores } \\
\text { de Juego de Tronos }\end{array}$ & Positivo \\
\hline El Mundo & $\begin{array}{l}\text { Así disfrutan los actores de Juego } \\
\text { de tronos de su nueva vida en Sevilla }\end{array}$ & Positivo \\
\hline Público & Los actores de Juego de Tronos en el Sevilla-Barça & Positivo \\
\hline El Español & Los actores de Juego de Tronos, sevillistas por un día & Positivo \\
\hline$A B C$ & Los actores de Juego de Tronos se hacen del Sevilla FC & Positivo \\
\hline El País & $\begin{array}{l}\text { Así de bien se lo pasaron los actores } \\
\text { de Juego de Tronos en el Sevilla-Barça }\end{array}$ & Positivo \\
\hline Eldiario.es & Juego de tronos es del Sevilla FC & Positivo \\
\hline 20 Minutos & $\begin{array}{l}\text { El elenco de Juego de Tronos, en el palco } \\
\text { del Pizjuán para ver el Sevilla-Barcelona }\end{array}$ & Positivo \\
\hline El Periódico & $\begin{array}{l}\text { Los actores de Juego de tronos animaron } \\
\text { al Sevilla en el duelo con el Barça }\end{array}$ & Positivo \\
\hline
\end{tabular}

Tabla 2. Resultados de noticias halladas en prensa, revistas y en páginas especializadas.

\begin{tabular}{|l|c|c|}
\hline \multicolumn{1}{|c|}{ Diarios } & Titular & $\begin{array}{c}\text { Impacto en la } \\
\text { imagen del club }\end{array}$ \\
\hline El Desmarque & $\begin{array}{r}\text { El recuerdo imborrable de Nervión } \\
\text { en Juego de Tronos }\end{array}$ & Positivo \\
\hline Fotogramas & $\begin{array}{r}\text { Los protagonistas de juego de tronos } \\
\text { se hacen forofos del Sevilla FC }\end{array}$ & Positivo \\
\hline Sport & Juego de Tronos, contra el Barça & Neutro \\
\hline As & $\begin{array}{r}\text { Los actores de Juegos de Tronos } \\
\text { disfrutaron del Sevilla-Barça }\end{array}$ & Positivo \\
\hline Marca & $\begin{array}{r}\text { Tyrion, Daenerys, la Montaña, Jaime Lannister, } \\
\text { Varys... jtodos se hacen del Sevilla! }\end{array}$ & Positivo \\
\hline Estadio Deportivo & $\begin{array}{r}\text { Juego de Tronos conquista el Sánchez-Pizjuán } \\
\text { Los protagonistas de Juego }\end{array}$ & Positivo \\
\hline Mundo deportivo & de Tronos, en el Sánchez Pizjuán & Positivo \\
\hline ecartelera.com & $\begin{array}{r}\text { El reparto de Juego de Tronos apoya al Sevilla } \\
\text { F.C. en su partido contra el Barça }\end{array}$ & Positivo \\
\hline Sportyou & Los actores de Juego de Tronos en el Pizjuán & Positivo \\
\hline Superdeporte & Los actores de Juego de Tronos, en el fútbol & Positivo \\
\hline
\end{tabular}




\begin{tabular}{|l|c|c|}
\hline Culturaocio.com & $\begin{array}{c}\text { Así vibraron las estrellas de Juego } \\
\text { de Tronos con el Sevilla-Barça }\end{array}$ & Positivo \\
\hline $\begin{array}{l}\text { La jugada } \\
\text { financiera }\end{array}$ & $\begin{array}{r}\text { La marca Sevilla F.C. crece a nivel internacional } \\
\text { gracias a Juego de Tronos }\end{array}$ & Positivo \\
\hline 90 min & $\begin{array}{r}\text { Los actores de Juego de Tronos disfrutan del } \\
\text { Sevilla FC - FC Barcelona en el Pizjuán }\end{array}$ & Positivo \\
\hline 35 milimetros & $\begin{array}{r}\text { Los actores de Juego de Tronos animan al Sevilla } \\
\text { FC en su último partido }\end{array}$ & Positivo \\
\hline Fórmula Tv & $\begin{array}{r}\text { Los actores de Juego de Tronos disfrutan del } \\
\text { fútbol durante su estancia en España }\end{array}$ & Positivo \\
\hline Hobby Consolas & $\begin{array}{r}\text { Juego de tronos-Tyrion-Jaime-Daenerys y otros } \\
\text { protagonistas en el Sevilla-barca }\end{array}$ & Positivo \\
\hline $\begin{array}{l}\text { Futbolsapiens. } \\
\text { com }\end{array}$ & $\begin{array}{r}\text { El Sánchez Pizjuán tuvo un Juego de Tronos } \\
\text { ze hicieron sevillistas }\end{array}$ & Positivo \\
\hline zeleb.es & $\begin{array}{r}\text { Juego de Tronos: ¿De qué equipo } \\
\text { son los Lannister? ¿Y los Stark...? }\end{array}$ & Positivo \\
\hline Sport & $\begin{array}{r}\text { El elenco de Juego de Tronos se hace } \\
\text { del Sevilla en el partido ante el Barça }\end{array}$ & Positivo \\
\hline La información & Positivo \\
\hline
\end{tabular}

Fuente: Elaboración propia, 2017.

Tabla 3. Resultados de noticias publicadas en diarios regionales.

\begin{tabular}{|c|c|c|}
\hline Diarios & Titular & $\begin{array}{l}\text { Impacto en la } \\
\text { imagen del club }\end{array}$ \\
\hline Diario de Sevilla & Una visita de tronío & Positivo \\
\hline Diario Vasco & $\begin{array}{l}\text { Juego de tronos: Los actores de Juego de } \\
\text { tronos, espectadores de lujo en el Pizjuán }\end{array}$ & Positivo \\
\hline Sevilla Actualidad & Sevilla, equipo de Los Siete Reinos & Positivo \\
\hline El norte de Castilla & Juegos de tronos conquista el Sánchez Pizjuán & Positivo \\
\hline La voz de Asturias & Juego de Tronos en el Sevilla-Barcelona & Positivo \\
\hline $\begin{array}{l}\text { La Opinión de } \\
\text { Tenerife }\end{array}$ & Los actores de Juego de Tronos, en el fútbol & Positivo \\
\hline Información & Los actores de Juego de Tronos, en el fútbol & Positivo \\
\hline Diario de Mallorca & Los actores de Juego de Tronos, en el fútbol & Positivo \\
\hline Diario Córdoba & $\begin{array}{l}\text { Los actores de Juego de tronos animaron al } \\
\text { Sevilla en el duelo con el Barça }\end{array}$ & Positivo \\
\hline Huelvahoy.com & $\begin{array}{l}\text { Los protagonistas de Juego } \\
\text { de Tronos, se hacen del Sevilla }\end{array}$ & Positivo \\
\hline La Nueva España & $\begin{array}{l}\text { Los actores de Juego de tronos revolucionan } \\
\text { Sevilla y también a los hinchas sevillistas }\end{array}$ & Positivo \\
\hline
\end{tabular}


Tabla 4. Resultados de publicaciones difundidas en portales de noticias de radio y televisión

\begin{tabular}{|l|c|c|}
\hline \multicolumn{1}{|c|}{ Diarios } & Titular & $\begin{array}{c}\text { Impacto en la } \\
\text { imagen del club }\end{array}$ \\
\hline Cadena Ser & $\begin{array}{c}\text { Los actores de Juego de Tronos se } \\
\text { comportaban como forofos del Sevilla }\end{array}$ & Positivo \\
\hline RTVE & Messi desactiva al Sevilla en un duelo de altura & Neutro \\
\hline Antena 3 & $\begin{array}{l}\text { El elenco de Juego de Tronos apoya } \\
\text { al Sevilla en su partido contra el Barça }\end{array}$ & Positivo \\
\hline Cuatro & $\begin{array}{r}\text { iJuego de Tronos es del Sevilla! Los actores } \\
\text { ven el partido en el Sánchez-Pizjuán }\end{array}$ & Positivo \\
\hline Radio Marca & $\begin{array}{r}\text { Los personajes de Juego de Tronos están } \\
\text { presentes en el Sánchez Pizjuán }\end{array}$ & Positivo \\
\hline Los40.com & $\begin{array}{r}\text { Los mejores memes de los actores de } \\
\text { juego de tronos en el Sevilla Barça }\end{array}$ & Positivo \\
\hline Antena 3 & $\begin{array}{l}\text { Los actores de Juego de Tronos revolucionan } \\
\text { las redes al asistir al partido del Sevilla-Barça }\end{array}$ & Positivo \\
\hline
\end{tabular}

Tabla 5. Resultados de Categorías de Contenido publicadas por el Sevilla Fútbol Club en Instagram y Twitter.

\begin{tabular}{|l|c|c|}
\hline \multicolumn{1}{|c|}{ Categoría de Contenido } & Instagram & Twitter \\
\hline Juego de Tronos & 1 & 8 \\
\hline Institucional & 0 & 7 \\
\hline Agenda & 0 & 9 \\
\hline Competición & 1 & 28 \\
\hline Jugadores & 1 & 6 \\
\hline Cantera & 0 & 2 \\
\hline Rueda de Prensa & 0 & 10 \\
\hline Entrevistas & 0 & 1 \\
\hline Afición & 0 & 1 \\
\hline Patrocinadores & 1 & 9 \\
\hline Otros medios & 0 & 12 \\
\hline Retweet & Sin datos & 0 \\
\hline
\end{tabular}

Fuente: Elaboración propia, 2017.

En cada tabla se han analizado las siguientes noticias:

Prensa generalista: 11

Prensa, revistas y páginas especializadas: 20

Prensa regional: 11

Radio y televisión: 7 
Total: 49

A modo de resumen, los diferentes impactos obtenidos por el club, recogidos a través de las fuentes consultadas, se han agrupado de la siguiente manera:

Impactos positivos: 47

Impactos neutros: 2

Impactos negativos: 0

Impactos totales: 49

\subsection{Contenidos redes sociales}

Por otro lado, en relación a las redes sociales del club analizadas, se han obtenido los siguientes resultados (ver tabla 5).

\section{Conclusiones}

La acción de comunicación desarrollada por el Sevilla Fútbol Club, S.A.D. en la prensa, permitió al equipo andaluz proyectar una imagen favorable ante sus diferentes públicos, especialmente en los medios de comunicación. Los resultados reflejan que en una muestra compuesta por 49 publicaciones, entre los diversos medios analizados, 47 noticias arrojan una imagen positiva hacia el club de fútbol, con un $96 \%$ de impactos obtenidos. Se observa que en dicha muestra no han existido impactos negativos que comprometieran o perjudicaran la imagen del club.

Por lo tanto, la gestión de esta acción puede considerarse correcta en términos comunicativos, teniendo en cuenta que se generaron impactos favorables en la prensa, permitiendo al equipo andaluz promocionarse y convertirse en noticia ante un buen número de empresas informativas $\mathrm{y}$, por ende, ante sus respectivas audiencias. Los contenidos publicados tuvieron un impacto favorable en la imagen corporativa del Sevilla, mejorando su reputación en el entorno online y confirmando la hipótesis planteada en el estudio.

Las redes sociales se configuran como una herramienta fundamental de las organizaciones deportivas, y en especial la red social Twitter, en lo que respecta a la emisión de contenidos producidos desde el club de fútbol. Se observa, en el análisis cualitativo ejecutado en la prensa, que en una gran parte de las noticias analizadas se recogen los tweets y las fotografías difundidas por el club a través de Twitter, que ha sido la red social seleccionada por el equipo de fútbol para darle una mayor cobertura al evento.

Además, se observa en la red social Twitter, que existe un reparto de categorías de contenido más heterogéneo que en Instagram, en lo que respecta al rol desempeñado por el club andaluz como emisor de contenidos variados en dichos medios.

La cuenta oficial en Twitter del Sevilla tuvo una mayor emisión de cantidad de contenido que su cuenta oficial en Instagram, pues en la primera se realizaron 93 publicaciones en total, 8 sobre Juego de Tronos, mientras que en la segunda apenas se realizaron publicaciones en las fechas en las que se realizó el estudio, con tan solo 4 publicaciones, de las que solamente una publicación fue sobre la serie.

El contenido relacionado con Juego de Tronos tuvo un peso destacado en las acciones comunicativas realizadas por el Sevilla en las redes sociales, aunque podría 
ser mayor en cuanto a la importancia dada con respecto a otros contenidos de otra temática que se han emitido, pues las publicaciones sobre la serie han tenido un porcentaje sobre el total que podría ser mayor, concretamente un $8,6 \%$ en Twitter, y un $25 \%$ en Instagram.

Se ha observado que el club de fútbol analizado ha centrado su manera de gestionar la comunicación en sus medios propios. Además, se ha observado, coincidiendo con García (2009), Paniagua, Gómez y Fernández-Sande (2012), que el emisor de contenido, en este caso el club de fútbol, utiliza las redes sociales Instagram y Twitter, más como canales de información, que de comunicación, pues no se aprovechan por completo las posibilidades que ofrecen dichos medios para interactuar con sus seguidores.

Además de publicar contenidos relacionados con la visita, se difundieron otras informaciones relevantes para el club, aprovechando el protagonismo creado en la prensa. Estos contenidos, clasificados en categorías, han servido para proyectar una imagen favorable del club, especialmente, a través de las redes sociales.

En lo que respecta a futuras líneas de investigación, teniendo en cuenta la clasificación de las áreas de comunicación de Berganza y Ruiz (2005) y según los resultados y limitaciones de este estudio, se podrían crear nuevas investigaciones centradas en los seguidores de un club de fútbol o en relación a los efectos sobre los mismos, así como contenido generado por el club de fútbol en sus cuentas oficiales en las redes sociales.

\section{Referencias bibliográficas}

Alonso, G., \& Avalos, J. (2013). La investigación del fútbol y sus nexos con los estudios de comunicación. Aproximaciones y ejemplos. Nueva Época, 20 (juliodiciembre), 33-64.

Andréu, J. (1998). Las técnicas del análisis de contenido: una revisión actualizada. Recuperado de http://bit.ly/19kKyGH

BBC Mundo (2017). 6 records que batió la serie "Game of Thrones", cuya séptima temporada se estrenó este domingo. En bbc.com. Publicado el 16/07/2017. Recuperado de http://www.bbc.com/mundo/noticias-40616313

Berganza, R., \& Ruiz, J. A. (2005). Investigar en Comunicación. Madrid: McGraw-Hill.

Cancelo, M., \& Almansa, A. (2013). Estrategias comunicativas en redes sociales. Estudio comparativo entre las Universidades de España y México. Historia y Comunicación Social, 18,423-435.doi: https://doi.org/10.5209/ rev_HICS.2013. v18.44339

Cano, R. (2017). Técnicas de marketing de los clubes de fútbol de élite en las redes sociales. Retos, 13, 43-58.

Cano, R. (2018). Comunicación digital en el mundo del fútbol (Tesis doctoral internacional). Universidad de Cádiz, Jerez de la Frontera.

Capriotti, P. (1999). Planificación estratégica de la imagen corporativa. Málaga: Instituto de Investigación en Relaciones Públicas. 
Capriotti, P. (2009). Branding corporativo. Santiago de Chile: Libros de la Empresa. Castillo, A. (2010). Introducción a las Relaciones Públicas. Recuperado de http:// bit.ly/2moPL7X

Castillo, A., Fernández, M., \& Castillero, E. (2016). Fútbol y redes sociales. Análisis de la gestión de Relaciones Públicas 2.0 por los clubes de fútbol. Estudios sobre el Mensaje Periodístico, 22(1), 239- 254.

Cole, J. (2016). Examining Facebook Practice: The Case of New Zealand Provincial Rugby. Massey University, Palmerston North, New Zealand.

Coutinho, E., \& Luzzi, A. (2017). Sports Marketing Plan: An Alternative Framework for Sports Club. International Journal of Marketing Studies; 9(4), 15-28. doi: http://doi.org/10.5539/ijms.v9n4p15

De Casas, P., Rodríguez, F. M., \& Aguaded, I. (2017). El discurso mediático en la prensa digital del baloncesto masculino y femenino en Huelva. SPORT TK: Revista Euroamericana de Ciencias del Deporte, 6, 147-156.

Domínguez, S., Álvarez, M., \& Martí, D. (2012). Dirección de comunicación en Internet. Estudio y recomendaciones para los espacios de prensa en webs corporativas desde el análisis de portales en internet de grupos empresariales en Galicia. Revista Internacional de Relaciones Públicas, 2(3), 45-70. doi: https:// doi.org/10.5783/RIRP-3-2012-03-45-70

Favaretto, C. (2014). La evolución de los ciberdiarios deportivos en el escenario de la web 2.0. El caso español (AS, Marca, Mundo Deportivo y Sport) (Tesis Doctoral). Universidad Autónoma de Barcelona, Barcelona. Recuperado de https://goo.gl/zIhDtG

Igartua, J. J., \& Humanes, M. L. (2004). Teoría e investigación en comunicación social. Madrid: Síntesis. En M. S. Valles, (2000): Técnicas cualitativas de investigación social. Madrid: Síntesis.

Lobillo, G., \& Muñoz, A. (2016). El papel de las redes sociales en las estrategias de expansión de mercado en los clubes de fútbol españoles. El uso personalizado de Twitter en lengua árabe del Real Madrid CF y FC Barcelona. Trípodos, 38, 195-214.

Olabe, F. (2010). La gestión de la comunicación corporativa en los clubes profesionales en España. Observatorio (OBS*), 3(3), 92-101.

Olabe, F. (2012). La comunicación digital del FC Barcelona y el Real Madrid CF y su percepción por los periodistas deportivos. Revista Internacional de Relaciones Públicas, 2(4), 277-298.

Micaletto, J. P., \& Gallardo, L. (2015). La comunicación institucional en la crisis del ébola en Europa: el caso de la crisis española de 2014 en sus inicios. Revista Internacional de Relaciones Públicas, 5(9), 89-110. Recuperado de http://bit. ly/2ktSlge

Moragas, M. (2003). El impacto de internet en los medios de comunicación y la industria del deporte. Centro de Estudios Olímpicos (CEO-UAB): Bellaterra. Recuperado de https://goo.gl/QkYJdB

Paché, G., \& Ika, L. (2017). European Professional Football Club Image and Human Capital Management. International Journal of Business Administration and Management Research, 2(3) Jul-Sep, 30-34.

Rojas, J. L.; Panal, A. (2017). El uso de Instagram en los medios de comunicación deportivos. Análisis comparado de Bleacher Report, L'équipe y Marca. Ámbitos, 38, Recuperado de http://institucional.us.es/ambitos/?p=3123 
Sotelo, J. (2012). Deporte y social media: el caso de la Primera División del fútbol español. Historia y comunicación social, 17, 217-230. doi: https://doi.org/10.5209/ rev_HICS.2012.v17.40607

Valles, M. (2000). Técnicas cualitativas de investigación social. Madrid: Síntesis.

Villafañe, J. (2005). La gestión de los intangibles empresariales. Comunicação e Sociedade, 8, 101-113.

Wilcox, D., Cameron, G., \& Xifra, J. (2009). Relaciones Públicas. Estrategias y tácticas. Madrid: Pearson Educación, S. A. 